\title{
Supporting Information for Temperature Dependence of HD Spin-Spin Coupling Constants of Heavy Metal Hydride and Dihydrogen Complexes Calculated by Vibrational Averaging
}

\author{
Brendan C. Mort and Jochen Autschbach* \\ Department of Chemistry, University at Buffalo, \\ State University of New York, Buffalo, New York 14260-3000 \\ email: jochena@buffalo.edu
}

\section{Vibrational Averaging Procedure}

In order to calculate the vibrational average of a molecular property at a specific temperature we begin by considering the vibrational states of a polyatomic molecule in the harmonic approximation, given as a set of quantum number vectors $\mathbf{v}$,

$$
\mathbf{v}=\left|v_{1}, v_{2}, v_{3}, \ldots, v_{3 n-6}\right\rangle
$$

where $v_{i}$ is the quantum number for harmonic oscillator number $i$. The vibrational energy $E_{\mathbf{v}}$ for state $\mathbf{v}$ is

$$
E_{\mathbf{v}}=\sum_{i} v_{i} \varepsilon_{i}
$$

where we have chosen, for convenience, the zero of the energy scale to be the zero-point vibrational energy $E_{\mathbf{0}}$. Further, $\varepsilon_{i}=\omega_{i} \hbar$. The partition function is

$$
Q=\sum_{\mathbf{v} \in V} e^{-E_{\mathbf{v}} / k T} .
$$

The equation for calculating the property $P$ at a temperature $T$ as a Boltzmann average of the property vibrationally averaged over each state $\mathbf{v}$ is then

$$
\langle P\rangle_{T}=\frac{1}{Q} \sum_{\mathbf{v} \in V}\langle P\rangle_{\mathbf{v}} e^{-E_{\mathbf{v}} / k T} .
$$

The vibrational average $\langle P\rangle_{\mathbf{v}}$ of a property $P$ for each vibrational state $\mathbf{v}$ can be shown to be given by Eq. (6) below. At this stage, anharmonicity corrections are included in the averaging process. 
The evaluation of Eq. (4) comes at negligible cost compared to the "data collection". Namely, in addition to the single point and spin-spin calculations at the equilibrium geometry, calculating the vibrational average of $J_{\mathrm{HD}}$ by numerical differentiation techniques requires the calculation of forces and spin-spin coupling constants for each one of 2(3n-6) geometries displaced from the equilibrium along the normal modes in order to determine the cubic force constants and the first and second property derivatives along the normal modes. ${ }^{1}$ Although this task involves a significant amount of computational resources, large commodity-type clusters have allowed for this task to be easily parallelized and the results can be obtained within an acceptable real-time frame.

A simple automated procedure for zero-point vibrational averaging of molecular properties has been described recently by the authors. ${ }^{1}$ For related work by other authors we refer to references cited in our previous paper. For the present work, we have expanded our implementation to include vibrational states beyond the zero-point. Let the nuclear vibrational wave function be represented initially by a product of harmonic-oscillator wave functions $\phi$ for each normal coordinate $q_{i}$.

$$
\psi_{\mathbf{v}}=\prod_{i} \phi_{i}^{v_{i}}\left(q_{i}\right)
$$

This vibrational wavefunction is the space-representation of the vector (1). Next, the cubic anharmonicity of the potential is considered to calculate a first-order perturbed vibrational wavefunction. The property $P$ is expanded around the equilibrium geometry as $P=P_{e}+\sum_{i}\left(\frac{\partial P}{\partial q_{i}}\right) q_{i}+$ $\frac{1}{2} \sum_{i j} \frac{\partial^{2} P}{\partial q_{i} \partial q_{j}} q_{i} q_{j}$. By using tabulated integrals ${ }^{2}$ involving the functions $\phi_{i}$ the property $P$ (in the present case, $J_{\mathrm{HD}}$ ) averaged over the perturbed vibrational state $\mathbf{v}$ is then, in atomic units, given as

$$
\begin{aligned}
\langle P\rangle_{\mathbf{v}} & =P_{e} \\
& +\frac{1}{2} \sum_{i}\left(\frac{\partial^{2} P}{\partial q_{i}^{2}}\right)\left(v_{i}+\frac{1}{2}\right) \\
& -\frac{1}{2} \sum_{i} \frac{1}{\omega_{i}}\left(\frac{\partial P}{\partial q_{i}}\right)\left(v_{i}+\frac{1}{2}\right) k_{i i i}-\frac{1}{2} \sum_{i} \frac{1}{\omega_{i}}\left(\frac{\partial P}{\partial q_{i}}\right) \sum_{j \neq i}\left(v_{j}+\frac{1}{2}\right) k_{i j j},
\end{aligned}
$$

where $P_{e}$ is the equilibrium value of the property, $k_{i i i}$ and $k_{i j j}$ are the cubic (anharmonic) force constants, $\omega_{i}$ is the frequency of vibrational transition $i$, and $q_{i}$ are the reduced normal coordinates. The vibrational average includes the PES anharmonicity and the property curvature perturbationally to first order. For the case when $\mathbf{v}=|0,0, \ldots, 0\rangle$, the vibrational averaging equation given above yields the zero-point vibrational average, which is given by

$$
\langle P\rangle_{\mathbf{0}}=P_{e}+\frac{1}{4} \sum_{i}\left(\frac{\partial^{2} P}{\partial q_{i}^{2}}\right)-\frac{1}{4} \sum_{i} \frac{1}{\omega_{i}}\left(\frac{\partial P}{\partial q_{i}}\right) \sum_{j} k_{i j j},
$$

in atomic units using reduced normal coordinates. The vibrationally averaged H-D distance is calculated from the anharmonic terms of equation (6). Equation (7) has been previously used by other authors ${ }^{3}$ and by us for zero-point vibrational averaging.

Because of the orthogonality of the harmonic oscillator functions $\phi_{i}$, by using Eq. (7) the 
temperature-dependent sum over all product states in Eq. (4) reduces to a simple compact form:

$$
\langle P\rangle_{T}=P_{e}+\frac{1}{4} \sum_{i} \operatorname{coth}\left(\frac{\varepsilon_{i}}{2 k T}\right)\left(\frac{\partial^{2} P}{\partial q_{i}^{2}}\right)-\frac{1}{4} \sum_{i} \frac{1}{\omega_{i}}\left(\frac{\partial P}{\partial q_{i}}\right) \sum_{j} \operatorname{coth}\left(\frac{\varepsilon_{j}}{2 k T}\right) k_{i j j}
$$

The first term in the vibrational correction involving the second derivatives of $P$ represents the property curvature correction $\Delta P_{p}$ in the main paper, the second term involving the cubic force constants $k_{i j j}$ represents the anharmonicity correction $\Delta P_{a}$. [After submission of our manuscript, a paper appeared where the property curvature contribution was presented in the same form. $\left.{ }^{4}\right]$ For each normal mode, the temperature-dependent factor has the behavior as shown in the figure below. For large $T$, the increase of the various individual contributions is approximately linear. For $T \rightarrow 0$ the coth factor approaches one and Eq. (8) reduces to Eq. (7).

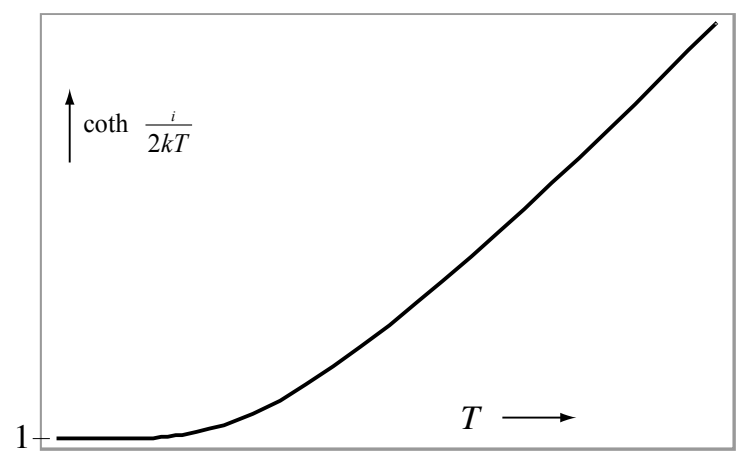

\section{Additional computational data for complex 1}

Table S1: Temperature dependence of the results for isomer 1a (cis-dihydride structure of $\mathbf{1}$ ) using the 6-31G(p) basis set for optimization and the 6-31G(p) basis set for calculation of $J_{\mathrm{HD}} . \Delta r_{\mathrm{HD}}$ is the vibrational correction to the equilibrium $\mathrm{H}-\mathrm{D}$ distance. $\left\langle r_{\mathrm{HD}}\right\rangle$ is the vibrational average of the H-D distance at the specified temperature. The vibrational correction to the HD spin-spin coupling constant is indicated by $\Delta J$. The correction consists of two components - an anharmonic correction $\left(\Delta J_{a}\right)$ and a property curvature correction $\left(\Delta J_{p}\right)$. The vibrational average of the HD spin-spin coupling constant is denoted by $\langle J\rangle$. See the discussion for more detail. The B3LYP functional was used for all calculations presented here. $J_{e}$ is $4.38 \mathrm{~Hz}$. The data marked with an asterisk have the contribution from normal mode \# $1\left(22 \mathrm{~cm}^{-1}\right)$ removed $\left(\mathrm{Cp}^{*}\right.$ hindered rotation).

\begin{tabular}{rrrrrrrr}
\hline & $0 \mathrm{~K}$ & $20 \mathrm{~K}$ & $100 \mathrm{~K}$ & $200 \mathrm{~K}$ & $300 \mathrm{~K}$ & $400 \mathrm{~K}$ & $600 \mathrm{~K}$ \\
\hline \hline$\Delta r_{\mathrm{HD}}(\AA)$ & -0.048 & -0.049 & -0.063 & -0.085 & -0.111 & -0.139 & -0.199 \\
$\left\langle r_{\mathrm{HD}}\right\rangle(\AA)$ & 1.604 & 1.603 & 1.589 & 1.567 & 1.541 & 1.513 & 1.453 \\
$\left\langle r_{\mathrm{HD}}\right\rangle^{*}(\AA)$ & 1.603 & 1.602 & 1.585 & 1.558 & 1.528 & 1.495 & 1.426 \\
$\Delta J_{a}(\mathrm{~Hz})$ & 0.584 & 0.584 & 0.495 & 0.351 & 0.217 & 0.098 & -0.108 \\
$\Delta J_{p}(\mathrm{~Hz})$ & 0.623 & 0.622 & 0.576 & 0.516 & 0.484 & 0.480 & 0.517 \\
$\Delta J(\mathrm{~Hz})$ & 1.207 & 1.206 & 1.072 & 0.867 & 0.701 & 0.577 & 0.409 \\
$\langle J\rangle(\mathrm{Hz})$ & 5.59 & 5.59 & 5.45 & 5.25 & 5.08 & 4.96 & 4.79 \\
$\langle J\rangle^{*}(\mathrm{~Hz})$ & 5.63 & 5.63 & 5.61 & 5.56 & 5.56 & 5.60 & 5.77 \\
\hline
\end{tabular}


Table S2: Temperature dependence of the results for isomer 1a using the IGLO-III basis set for optimization as well as calculation of $J_{\mathrm{HD}}$. For further details see caption of Table $\mathrm{S} 1$. All calculations were performed with the B3LYP functional. $J_{e}$ is $4.36 \mathrm{~Hz}$. The data marked with an asterisk have the contribution from normal mode \# $1\left(21 \mathrm{~cm}^{-1}\right)$ removed $\left(\mathrm{Cp}^{*}\right.$ hindered rotation).

\begin{tabular}{rrrrrrrr}
\hline & $0 \mathrm{~K}$ & $20 \mathrm{~K}$ & $100 \mathrm{~K}$ & $200 \mathrm{~K}$ & $300 \mathrm{~K}$ & $400 \mathrm{~K}$ & $600 \mathrm{~K}$ \\
\hline \hline$\Delta r_{\mathrm{HD}}(\AA)$ & -0.066 & -0.068 & -0.096 & -0.140 & -0.190 & -0.242 & -0.351 \\
$\left\langle r_{\mathrm{HD}}\right\rangle(\AA)$ & 1.584 & 1.582 & 1.554 & 1.510 & 1.460 & 1.408 & 1.299 \\
$\left\langle r_{\mathrm{HD}}\right\rangle^{*}(\AA)$ & 1.580 & 1.579 & 1.548 & 1.498 & 1.444 & 1.386 & 1.267 \\
$\Delta J_{a}(\mathrm{~Hz})$ & 0.860 & 0.870 & 1.036 & 1.279 & 1.549 & 1.843 & 2.473 \\
$\Delta J_{p}(\mathrm{~Hz})$ & 0.663 & 0.663 & 0.640 & 0.617 & 0.627 & 0.664 & 0.788 \\
$\Delta J(\mathrm{~Hz})$ & 1.523 & 1.533 & 1.676 & 1.896 & 2.176 & 2.507 & 3.260 \\
$\langle J\rangle(\mathrm{Hz})$ & 5.88 & 5.89 & 6.03 & 6.26 & 6.53 & 6.87 & 7.62 \\
$\langle J\rangle^{*}(\mathrm{~Hz})$ & 6.04 & 6.06 & 6.32 & 6.75 & 7.24 & 7.79 & 8.99 \\
\hline
\end{tabular}

Table S3: Temperature dependence of the results for isomer $\mathbf{1 b}$ (dihydrogen structure of $\mathbf{1}$ ) using the 6-31G(p) basis set for both optimization and calculation of $J_{\mathrm{HD}}$. For further details see caption of Table S1. The B3LYP functional was used for all calculations presented here. $J_{e}$ is $30.45 \mathrm{~Hz}$.

\begin{tabular}{rrrrrrrr}
\hline & $0 \mathrm{~K}$ & $20 \mathrm{~K}$ & $100 \mathrm{~K}$ & $200 \mathrm{~K}$ & $300 \mathrm{~K}$ & $400 \mathrm{~K}$ & $600 \mathrm{~K}$ \\
\hline \hline$\Delta r_{\mathrm{HD}}(\AA)$ & 0.098 & 0.098 & 0.099 & 0.100 & 0.103 & 0.107 & 0.119 \\
$\left\langle r_{\mathrm{HD}}\right\rangle(\AA)$ & 1.037 & 1.037 & 1.038 & 1.039 & 1.042 & 1.046 & 1.058 \\
$\Delta J_{a}(\mathrm{~Hz})$ & -4.165 & -4.173 & -4.264 & -4.494 & -4.847 & -5.294 & -6.356 \\
$\Delta J_{p}(\mathrm{~Hz})$ & -0.712 & -0.711 & -0.697 & -0.723 & -0.824 & -0.977 & -1.359 \\
$\Delta J(\mathrm{~Hz})$ & -4.877 & -4.884 & -4.961 & -5.217 & -5.671 & -6.271 & -7.714 \\
$\langle J\rangle(\mathrm{Hz})$ & 25.58 & 25.57 & 25.49 & 25.24 & 24.78 & 24.18 & 22.74 \\
\hline
\end{tabular}

Table S4: Temperature dependence of the results for isomer $\mathbf{1 b}$ using the IGLO-III basis set for optimization and calculation of $J_{\mathrm{HD}}$. For further details see caption of Table $\mathrm{S} 1$. All calculations have been performed with the B3LYP functional. $J_{e}$ is $30.72 \mathrm{~Hz}$.

\begin{tabular}{rrrrrrrr}
\hline & $0 \mathrm{~K}$ & $20 \mathrm{~K}$ & $100 \mathrm{~K}$ & $200 \mathrm{~K}$ & $300 \mathrm{~K}$ & $400 \mathrm{~K}$ & $600 \mathrm{~K}$ \\
\hline \hline$\Delta r_{\mathrm{HD}}(\AA)$ & 0.053 & 0.053 & 0.055 & 0.056 & 0.057 & 0.058 & 0.061 \\
$\left\langle r_{\mathrm{HD}}\right\rangle(\AA)$ & 0.983 & 0.983 & 0.985 & 0.986 & 0.987 & 0.988 & 0.991 \\
$\Delta J_{a}(\mathrm{~Hz})$ & -0.726 & -0.736 & -0.902 & -1.210 & -1.564 & -1.937 & -2.700 \\
$\Delta J_{p}(\mathrm{~Hz})$ & -1.370 & -1.368 & -1.336 & -1.282 & -1.257 & -1.273 & -1.386 \\
$\Delta J(\mathrm{~Hz})$ & -2.095 & -2.104 & -2.238 & -2.492 & -2.821 & -3.210 & -4.086 \\
$\langle J\rangle(\mathrm{Hz})$ & 28.63 & 28.62 & 28.48 & 28.23 & 27.90 & 27.51 & 26.63 \\
\hline
\end{tabular}




\section{Additional data for complex 2}

Table S5: Temperature dependence of the results for complex $2(\mathrm{H}-\mathrm{H})$ using the 6-31G $(\mathrm{p})$ basis set for optimization and calculation of $J_{\mathrm{HH}}$. For further details see caption of Table $\mathrm{S} 1$. All calculations have been performed with the MPW1PW91 functional. $J_{e}$ is $2.37 \mathrm{~Hz}$ (in H-D units). All spinspin coupling constants reported here are in $J_{\mathrm{HD}}$ units to allow for an easy comparison with $J_{\mathrm{HD}}$. Multiply with 6.5144 to convert to $J_{\mathrm{HH}}$. The data marked with an asterisk have the contribution from normal mode \# $1\left(22 \mathrm{~cm}^{-1}\right)$ removed (Cp rotations).

\begin{tabular}{rrrrrrrr}
\hline & $0 \mathrm{~K}$ & $20 \mathrm{~K}$ & $100 \mathrm{~K}$ & $200 \mathrm{~K}$ & $300 \mathrm{~K}$ & $400 \mathrm{~K}$ & $600 \mathrm{~K}$ \\
\hline \hline$\Delta r_{\mathrm{HH}}(\AA)$ & -0.022 & -0.023 & -0.035 & -0.052 & -0.072 & -0.093 & -0.138 \\
$\left\langle r_{\mathrm{HH}}\right\rangle(\AA)$ & 1.684 & 1.683 & 1.671 & 1.654 & 1.634 & 1.613 & 1.568 \\
$\Delta J_{a}(\mathrm{~Hz})$ & 0.354 & 0.359 & 0.412 & 0.488 & 0.572 & 0.664 & 0.862 \\
$\Delta J_{p}(\mathrm{~Hz})$ & 0.433 & 0.444 & 0.553 & 0.701 & 0.862 & 1.037 & 1.417 \\
$\Delta J(\mathrm{~Hz})$ & 0.787 & 0.803 & 0.965 & 1.189 & 1.434 & 1.701 & 2.278 \\
$\langle J\rangle(\mathrm{Hz})$ & 3.16 & 3.17 & 3.33 & 3.56 & 3.80 & 4.07 & 4.65 \\
$\langle J\rangle^{*}(\mathrm{~Hz})$ & 3.14 & 3.14 & 3.21 & 3.30 & 3.42 & 3.56 & 3.89 \\
\hline
\end{tabular}

Table S6: Temperature dependence of the results for complex $2(\mathrm{H}-\mathrm{T})$ using the 6-31G(p) basis set for optimization and calculation of $J_{\mathrm{HT}}$. For further details see caption of Table $\mathrm{S} 1$. All calculations have been performed with the MPW1PW91 functional. $J_{e}$ is $2.37 \mathrm{~Hz}$ (in H-D units). All spinspin coupling constants reported here are in $J_{\mathrm{HD}}$ units to allow for an easy comparison with $J_{\mathrm{HD}}$. Multiply with 6.9486 to convert to $J_{\mathrm{HT}}$. The data marked with an asterisk have the contribution from normal mode \# $1\left(19 \mathrm{~cm}^{-1}\right)$ removed (Cp rotations).

\begin{tabular}{rrrrrrrr}
\hline & $0 \mathrm{~K}$ & $20 \mathrm{~K}$ & $100 \mathrm{~K}$ & $200 \mathrm{~K}$ & $300 \mathrm{~K}$ & $400 \mathrm{~K}$ & $600 \mathrm{~K}$ \\
\hline \hline$\Delta r_{\mathrm{HT}}(\AA)$ & -0.017 & -0.019 & -0.031 & -0.049 & -0.069 & -0.091 & -0.136 \\
$\left\langle r_{\mathrm{HT}}\right\rangle(\AA)$ & 1.689 & 1.687 & 1.675 & 1.657 & 1.637 & 1.615 & 1.570 \\
$\Delta J_{a}(\mathrm{~Hz})$ & 0.291 & 0.296 & 0.352 & 0.433 & 0.524 & 0.624 & 0.837 \\
$\Delta J_{p}(\mathrm{~Hz})$ & 0.345 & 0.356 & 0.465 & 0.618 & 0.789 & 0.974 & 1.367 \\
$\Delta J(\mathrm{~Hz})$ & 0.636 & 0.652 & 0.817 & 1.051 & 1.313 & 1.598 & 2.204 \\
$\langle J\rangle(\mathrm{Hz})$ & 3.01 & 3.02 & 3.19 & 3.42 & 3.68 & 3.97 & 4.57 \\
$\langle J\rangle^{*}(\mathrm{~Hz})$ & 3.00 & 3.00 & 3.07 & 3.18 & 3.31 & 3.47 & 3.82 \\
\hline
\end{tabular}




\section{Additional data for complex 6}

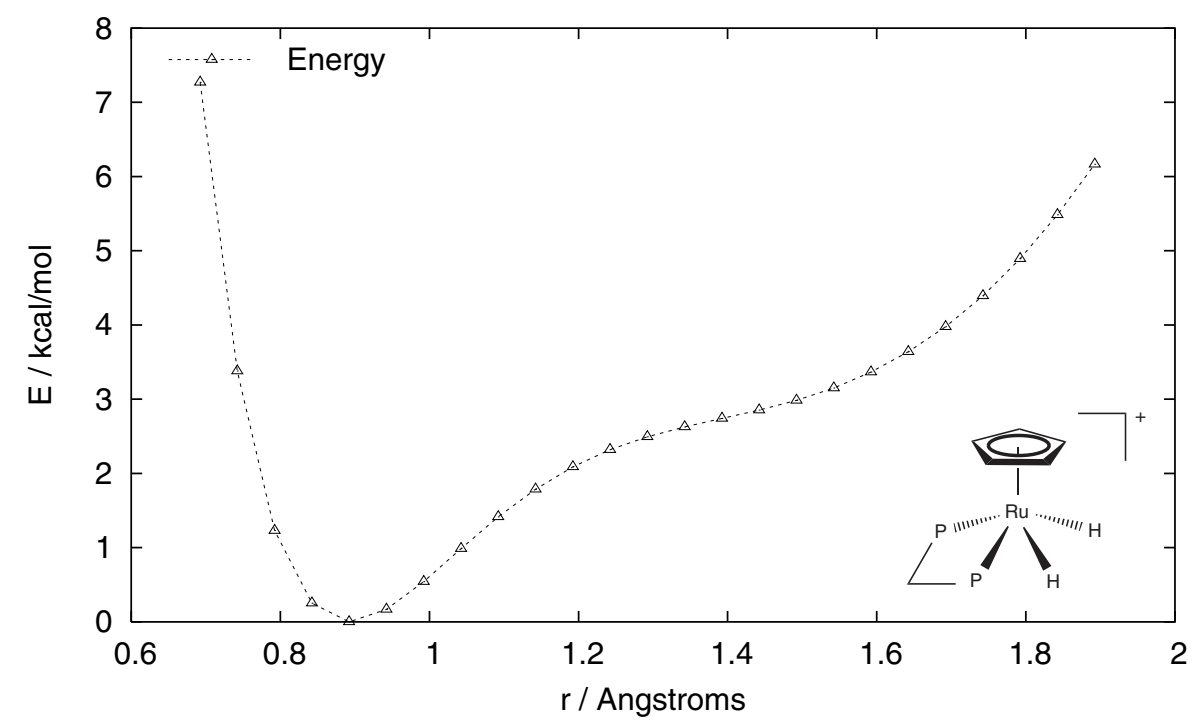

Figure S1: Energy of complex 6 as a function of $\mathrm{H}-\mathrm{H}$ internuclear distance using the IGLO-III basis set for the $\mathrm{H}$ atoms attached to $\mathrm{Ru}$. Each point of the relaxed PES scan has been optimized with the B3LYP functional.

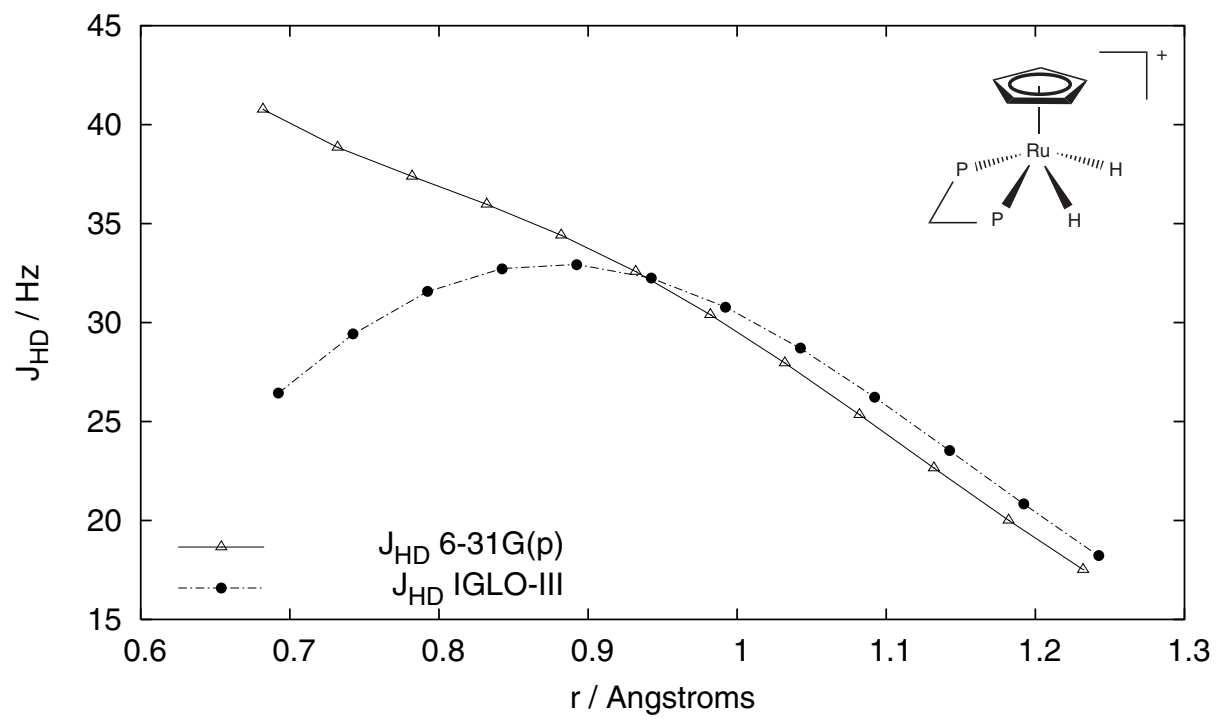

Figure S2: $J_{\mathrm{HD}}$ of complex 6 as a function of $\mathrm{H}-\mathrm{H}$ internuclear distance using the 6-31G(p) and the IGLO-III basis set for the $\mathrm{H}$ atoms attached to Ru. Each point of the relaxed PES scan has been optimized with the B3LYP functional. The spin-spin coupling constant has been calculated at each one of the relaxed PES geometries. The decrease of the IGLO-III coupling constant is not very intuitive at first sight. We attribute the different behavior of the basis sets partially to the fact that the IGLO-III basis covers a larger spatial range. 
Table S7: Temperature dependence of the results for complex 6 using the IGLO-III basis set for both optimization and for calculation of $J_{\mathrm{HD}}$. For further details of the notation, see Table 2. The B3LYP hybrid functional was used for all calculations presented here. $J_{e}$ is $32.94 \mathrm{~Hz}$. The data marked with an asterisk have the contribution from normal mode \# $1\left(44 \mathrm{~cm}^{-1}\right)$ removed (Cp rotations).

\begin{tabular}{rrrrrrrr}
\hline & $0 \mathrm{~K}$ & $20 \mathrm{~K}$ & $100 \mathrm{~K}$ & $200 \mathrm{~K}$ & $300 \mathrm{~K}$ & $400 \mathrm{~K}$ & $600 \mathrm{~K}$ \\
\hline \hline$\Delta r_{\mathrm{HD}}(\AA)$ & 0.013 & 0.013 & 0.012 & 0.007 & -0.001 & -0.010 & -0.031 \\
$\left\langle r_{\mathrm{HD}}\right\rangle(\AA)$ & 0.905 & 0.905 & 0.904 & 0.899 & 0.891 & 0.882 & 0.861 \\
$\Delta J_{a}(\mathrm{~Hz})$ & 0.189 & 0.187 & 0.146 & 0.046 & -0.091 & -0.238 & -0.528 \\
$\Delta J_{p}(\mathrm{~Hz})$ & -0.545 & -0.544 & -0.490 & -0.342 & -0.129 & 0.100 & 0.555 \\
$\Delta J(\mathrm{~Hz})$ & -0.357 & -0.357 & -0.344 & -0.295 & -0.220 & -0.138 & 0.027 \\
$\langle J\rangle(\mathrm{Hz})$ & 32.59 & 32.59 & 32.60 & 32.65 & 32.72 & 32.80 & 32.97 \\
$\langle J\rangle^{*}(\mathrm{~Hz})$ & 32.57 & 32.57 & 32.54 & 32.54 & 32.56 & 32.58 & 32.64 \\
\hline
\end{tabular}

\section{Complete References 23 and 30}

- Complete Reference 23:

Gaussian 03, Revision C.02, M. J. Frisch, G. W. Trucks, H. B. Schlegel, G. E. Scuseria, M. A. Robb, J. R. Cheeseman, J. A. Montgomery, Jr., T. Vreven, K. N. Kudin, J. C. Burant, J. M. Millam, S. S. Iyengar, J. Tomasi, V. Barone, B. Mennucci, M. Cossi, G. Scalmani, N. Rega, G. A. Petersson, H. Nakatsuji, M. Hada, M. Ehara, K. Toyota, R. Fukuda, J. Hasegawa, M. Ishida, T. Nakajima, Y. Honda, O. Kitao, H. Nakai, M. Klene, X. Li, J. E. Knox, H. P. Hratchian, J. B. Cross, C. Adamo, J. Jaramillo, R. Gomperts, R. E. Stratmann, O. Yazyev, A. J. Austin, R. Cammi, C. Pomelli, J. W. Ochterski, P. Y. Ayala, K. Morokuma, G. A. Voth, P. Salvador, J. J. Dannenberg, V. G. Zakrzewski, S. Dapprich, A. D. Daniels, M. C. Strain, O. Farkas, D. K. Malick, A. D. Rabuck, K. Raghavachari, J. B. Foresman, J. V. Ortiz, Q. Cui, A. G. Baboul, S. Clifford, J. Cioslowski, B. B. Stefanov, G. Liu, A. Liashenko, P. Piskorz, I. Komaromi, R. L. Martin, D. J. Fox, T. Keith, M. A. Al-Laham, C. Y. Peng, A. Nanayakkara, M. Challacombe, P. M. W. Gill, B. Johnson, W. Chen, M. W. Wong, C. Gonzalez, and J. A. Pople, Gaussian, Inc., Wallingford CT, 2004.

- Complete Reference 30:

Basis sets were obtained from the Extensible Computational Chemistry Environment Basis Set Database, Version 02/25/04, as developed and distributed by the Molecular Science Computing Facility, Environmental and Molecular Sciences Laboratory which is part of the Pacific Northwest Laboratory, P.O. Box 999, Richland, Washington 99352, USA, and funded by the U.S. Department of Energy. The Pacific Northwest Laboratory is a multi-program laboratory operated by Battelle Memorial Institute for the U.S. Department of Energy under contract DE-AC06-76RLO 1830. Contact Karen Schuchardt for further information. URL http://www.emsl.pnl.gov/forms/basisform.html

\section{References}

[1] Mort, B. C.; Autschbach, J. J. Phys. Chem. A 2005, 109, 8617-8623. 
[2] Wilson, E. B. Molecular Vibrations: The Theory of Infrared and Raman Vibrational Spectra; McGraw-Hill: New York, 1955.

[3] Sauer, S. P. A.; Packer, M. J. The ab initio calculation of molecular properties other than the potential energy surface. In Computational Molecular Spectroscopy; Bunker, P. R.; Jensen, P., Eds.; John Wiley \& Sons: London, 2000.

[4] Kongsted, J.; Pedersen, T. B.; Jensen, L.; Hansen, A. E.; Mikkelsen, K. V. J. Am. Chem. Soc. 2006, 128, 976-982. 